\title{
El derecho a la información y la regulación en el tratamiento de la COVID-19 en los países andinos
}

\author{
Abel SuIng \\ Universidad Técnica Particular \\ de Loja, Ecuador \\ arsuing@utpl.edu.ec \\ https://orcid.org/0000-0002-4234-5926
}

\author{
Lilia CarPio-JIMÉnez \\ Universidad Técnica Particular \\ de Loja, Ecuador \\ lkcarpio@utpl.edu.ec \\ https://orcid.org/0000-0002-3337-1647
}

\author{
Kruzkaya OrdóÑez \\ Universidad Técnica Particular \\ de Loja, Ecuador \\ kordonez@utpl.edu.ec \\ https://orcid.org/0000-0002-2360-8188
}

\section{The right to information and regulation in the treatment of COVID-19 in the Andean countries}

\begin{abstract}
RESUMEN
Las medidas que adoptaron los gobiernos andinos para menguar los contagios de COVID-19 generan nuevos escenarios. Algunas políticas afectan a los medios de comunicación, al trabajo de los periodistas y alertan sobre posibles abusos al restringirse algunas libertades. Cobra interés el examen de las autoridades de regulación de los países andinos porque, entre otros motivos, es la región de Latinoamérica donde la pandemia alcanzó rápidamente cifras altas y se desbordaron los sistemas de salud. La metodología es cualitativa de tipo descriptivo y relacional a través de análisis de contenidos a

1) las declaraciones de organizaciones y expertos internacionales, y las disposiciones de los gobiernos, 2) las acciones de los reguladores iberoamericanos, y 3) las publicaciones en medios sociales. Las recomendaciones que brindan las instituciones extranjeras coinciden con las premisas que motivaron la creación de los órganos de regulación. Las normas nacionales acogen los debates sobre pluralismo, diversidad y derecho a la comunicación, por lo tanto, no hay ausencias de precedentes jurídicos ni de estructuras públicas. En la construcción de equilibrios entre el control de la pandemia, la recuperación económica y el mantenimiento de las garantías lo urgente es poner en marcha los andamiajes diseñados con la participación de los ciudadanos.
\end{abstract}

PALABRAS CLAVE

Comunicación; COVID-19; Derecho a la información; Medios de comunicación; Políticas de comunicación; Regulación.

\section{ABSTRACT}

The measures adopted by the Andean governments to reduce COVID-19 infections generate new scenarios. Some policies affect the media, the work of journalists and warn of possible abuses as some freedoms are restricted. It is of interest to the regulatory authorities of the Andean countries because, among other reasons, this is the region of Latin America where the pandemic quickly reached high levels and health systems were overwhelmed. The methodology is qualitative, descriptive and relational through content analysis of 1) statements by international organisations and experts, and government provisions, 2) actions of Latin American regulators, and 3) publications in the social media. The recommendations provided by foreign institutions coincide with the premises that motivated the creation of the regulatory bodies. The national regulations welcome the debates on pluralism, diversity and the right to communication; therefore, there is no lack of legal precedent or public structures. In the construction of balances between controlling the pandemic, economic recovery and maintaining guarantees, the urgent thing is to put in place the scaffolding designed with the participation of citizens.

\section{KEYWORDS}

Communication; COVID-19; Right to information; Media; Communication policies; Regulation. los países andinos. Hipertext.net, (21), 27-40. https://doi.org/10.31009/hipertext.net.2020.i21.03 


\section{El dret a la informació i la regulació en el tractament de la COVID-19 en els països andis}

\section{RESUM}

Les mesures que els governs andins van disposar per minvar els contagis de COVID-19 generen nous escenaris entre els ciutadans. Algunes polítiques afecten els mitjans de comunicació, la feina dels periodistes i alerten sobre possibles abusos al restringir-se llibertats. Cobra interès examinar les autoritats de regulació dels països andins perquè, entre altres motius, fou la regió llatinoamericana on la pandèmia va assolir mes ràpidament xifres altes i on es van desbordar els sistemes de salut. S'ha utilitzat metodologia qualitativa de tipus descriptiu i relacional a través d'anàlisis de continguts a 1) les declaracions d'organitzacions i experts internacionals així com a les disposicions dels governs; 2) les accions de reguladors iberoamericans; i 3) les publicacions en mitjans socials. Les recomanacions que brinden les institucions estrangeres coincideixen amb les premisses que van motivar la creació dels òrgans de regulació. Les normes nacionals acullen els debats sobre pluralisme, diversitat i dret a la comunicació, per tant, no hi ha absències de precedents jurídics ni d'estructures públiques. En la construcció d'equilibris entre el control de la pandèmia, la recuperació econòmica i el manteniment de les garanties el que és urgent és posar en marxa ordenacions dissenyades amb la participació dels ciutadans.

\section{PARAULES CLAU}

Comunicació; COVID-19; Dret a la informació; Mitjans de comunicació; Polítiques de comunicació; Regulació.

\section{Introducción}

Los gobiernos controlan la expansión del COVID-19 a través de políticas sanitarias y la restricción a la libre circulación de las personas, entre otras acciones. En algunos países se dispuso la vigilancia digital de los contagiados, ejemplo de ello es Ecuador donde Lenin Moreno mediante Decreto Presidencial dispuso que podrían utilizarse plataformas satelitales y de telefonía móvil para ubicar a las personas, ante ello la Corte Constitucional del país andino condicionó el recurso a cuatro parámetros: utilizarlo en estado de excepción, no transgredir los derechos a la privacidad, asegurar la información personal de los pacientes, y realizar el seguimiento "a las personas que el Ministerio de Salud Pública haya identificado como portadores del virus" (Romero, 2020).

Contradictoriamente, esas operaciones de vigilancia no tienen sentido porque "los datos sobre las ubicaciones de las personas no son lo suficientemente precisos para el seguimiento automático de contactos" (Stanley y Granick, 2020).

Entre marzo y mayo de 2020 los ciudadanos vieron disminuidos algunos derechos, además el distanciamiento alejó "un espacio de intercambio necesario para la política (...) algunas partes de la democracia se traducen mal en un mundo en línea" (Jon, 2020). Las decisiones encaminadas a menguar los impactos de COVID-19 derivarán en totalitarismos si no ocurre la participación cívica de la población, incluso sobre el temor a la vigilancia o al usufructo de sus datos (Bedoya, 2020; Calo, 2020; Cetina, 2020; Véliz, 2020).

La libertad de expresión va de la mano de los derechos a la comunicación (OEA, 2020) y es celosamente protegida porque es un pivote de la democracia, de allí el interés en que los gobiernos cuiden que las medidas impuestas para salvaguardar a las personas sean específicas y se tomen solo por razones justificadas. La libertad de expresión y el libre flujo de información son requisitos imprescindibles para informar en y sobre la pandemia. "Medios, periodistas, organismos públicos de radiodifusión tienen un papel clave y una responsabilidad especial para proporcionar información precisa y confiable, pero también para prevenir el pánico y fomentar la cooperación" (Council of Europe, 2020, p. 6).

Los medios de comunicación "deben responder y garantizar la libertad de expresión y el derecho a la información en el contexto de la pandemia" (Bizberge, 2020), sin embargo ¿qué instituciones están encargadas de velar por la libertad de expresión? En principio son los sistemas de justicia a través de las cortes, también 
los parlamentos, pero específicamente son las autoridades de regulación o Consejos de Comunicación.

La información sobre salud es "sensible y, por ende, debe ser particularmente protegida (...) se presentan informaciones imprecisas, confusas y hasta contradictorias entre sí" (Segura, 2020, p. 56), por ello la comunicación masiva "debe constituir una herramienta formal de la salud pública para confrontar la amenaza del COVID-19, y una oportunidad de educación, aprendizaje y prevención ante futuros eventos de emergencia sanitaria" (Moreno-Montoya, 2020).

La comunicación a los ciudadanos evita incertidumbres y previene escenarios de violencia (Cortes, 2020; Ruvalcaba, et al., 2020), es clave para generar confianza en los gobiernos y trabajar colaborativamente en el manejo de la crisis (Blackman, 2020), esto supone "una gran inversión en salud pública" (Torres y Valenzuela, 2020), aunque también hay excesos a pretexto de luchar contra la desinformación, "gobiernos represivos que pretenden controlar la narrativa sobre la expansión de la enfermedad han intensificado la persecución contra periodistas" (Lauría, 2020, 39) o han adoptado medidas contra la prensa.

A partir de lo expuesto interesa conocer el desempeño de los órganos de regulación de comunicación de los países andinos (Bolivia, Colombia, Ecuador y Perú) frente al embate de la pandemia porque forman un bloque político y económico desde 1969 (CAN, 2020) y es donde están las primeras ciudades fuertemente afectadas en la región, Guayaquil y Piura, que vieron desbordadas sus capacidades para atender pacientes con COVID-19 (Cué, 2020; Torres, 2020).

En Guayaquil se llegó al punto de encontrar "cadáveres abandonados en las calles [otros] yacían en sus casas sin ser retirados por varios días" (Bajaña, 2020, p. 3). A partir de la crisis sanitaria vivida en Ecuador, Perú y Chile la Organización Mundial de la Salud "declaró a Latinoamérica como el nuevo epicentro de la pandemia" (Vassoler-Froelich, 2020, p. 6) en mayo de 2020. La COVID-19 mostró lo desamparado que estaba el sistema de salud (Evans, Sabucedo y Paz, 2020).

\section{Fundamentos}

El derecho a la información "está en el corazón de la democracia [...] si se implementa correctamente, permite un diálogo entre el público y sus gobernantes, cultiva la buena gobernanza y promueve la rendición de cuentas" (Mendel, 2009, p. 1), para su vigencia se han dispuesto normas y leyes sobre la base del artículo 19 de la Declaración Universal de los Derechos Humanos
(1948), aunque también corresponden a la Declaración de Derechos de Virginia (1776), génesis de la Constitución de Estados Unidos, y a la Declaración de los Derechos del Hombre y el Ciudadano de la Asamblea Nacional de Francia (1789), documentos donde se expresa la libertad de pensamiento y opinión como derechos de los ciudadanos.

En la práctica hay interdependencia entre la libertad de expresión, la libertad de información y el derecho a la información porque constituyen, por su naturaleza, presupuestos de una sociedad libre y democrática (Balaguer, 2013). El Informe Mac Bride (1980) impulsó el debate sobre los derechos asociados a la información y la comunicación, y en la segunda década del siglo XXI en América Latina se crean leyes y órganos de regulación para "democratizar" la comunicación.

Respecto al derecho a la comunicación Damián Loreti (2005) señala que:

Desde las propias discusiones sobre los alcances del derecho a la comunicación y sus solapamientos o enfrentamientos con el derecho a la información, así como la mismísima comprensión de este concepto, hemos ido transitando por los más diversos y sinuosos caminos, trayectos en los cuales nos cruzamos con la libertad de expresión, la libertad de prensa, el derecho de la información, el derecho a la información, el derecho de acceso a la información y otros varios actores.

La simbiosis de estos derechos "es producto de décadas de campañas, definición de estándares y jurisprudencia" (Mendel, 2009, p. 1) que evidencian el valor de la información como un derecho político de participación social y de carácter colectivo. Emitir información y recibirla son dos aspectos de un mismo derecho que aportan esencia a la libertad de expresión, en la medida en que son instrumentos necesarios para el ejercicio de otros derechos políticos de los ciudadanos (Carrillo, 1986). Como contrapeso hay límites al derecho de comunicar y expresar opiniones. Los límites tienen que ver con la protección de los derechos de niñas, niños y adolescentes; y con el sigilo en las áreas de infraestructura básica como salud, defensa y orden público.

Éstas premisas justifican la creación de las autoridades de regulación de la comunicación, además su existencia promueve el acceso a los medios y la diversidad (McQuail, 2010), deben ser independientes, operar de manera transparente, rendir cuentas al público y supervisar a los actores privados (The Centre for Law and Democracy, 2019). Los Consejos de Comunicación coadyuvan a la "convivencia de distintos puntos de vista políticos y culturales que es necesario garantizar a tra- 
vés de una intervención estatal" (Krakowiak, 2018, p. 125).

La gestión de los medios de comunicación y los organismos de regulación debe ser comprendida dentro de sistemas sociopolíticos amplios porque son parte activa de los mismos. Daniel C. Hallin y Paolo Mancini (2008) establecieron para Europa y Norteamérica tres grandes sistemas de interdependencia entre la organización sociopolítica y los medios de comunicación: liberal, democrático corporativo y pluralista polarizado. El modelo de América Latina es un híbrido entre el sistema liberal y el pluralista polarizado (Hallin y Mancini, 2007, p. 91).

El modelo liberal corresponde a los países anglosajones del Atlántico Norte, se caracteriza por brindar garantías al libre ejercicio de las empresas. Respeta el pluralismo de ideas y las orientaciones políticas de los ciudadanos. El Estado juega un papel de orientador y regulador, pero limitado a amparar una economía de libre mercado.

En los países del mediterráneo el sistema de medios configura un modelo pluralista polarizado, se identifica con una cultura tardía de la libertad de prensa, menor profesionalización y mayor vinculación político-partidista. El Estado desempeña el rol de propietario, promotor o regulador. Latinoamérica por su cercanía histórica con España y Portugal tiene características parecidas a este modelo.

La regulación en comunicación inicialmente se aplicó a la radiodifusión (Aguilar, 2016), y pretendió intervenir en cuestiones técnicas y de control de mercado (Pimiento, 2013). En América Latina desde la década de 1970 cambió el paradigma de vigilancia hacia el fomento del pluralismo y la democratización de la comunicación, en concreto de los derechos humanos (Hernández, Badillo y Peña, 2018; Loreti y Lozano, 2014). Hacia el primer cuarto del siglo XXI la premisa de libertad de expresión es "insuficiente para procesar los conflictos, necesidades y demandas contemporáneas relacionadas con la comunicación y el desarrollo de los pueblos en el marco de la democracia y el Estado de Derecho" (Jurado, 2010, p. 87).

El propósito de regular la comunicación en COVID-19 es lograr información oportuna, de acceso rápido, gratuita, verificada y de alta calidad para optimizar las acciones de ciudadanos, médicos y autoridades (Chan, Nickson, Rudolph, Lee y Joynt, 2020; Massaad y Cherfan, 2020; Viet-Phuong et al., 2020). Así mismo, los formuladores de políticas "deberían insistir en los esfuerzos por aislar las opiniones que se basan en noticias falsas" (Ahmed,
Vidal-Alaball, Downing y López, 2020).

\section{Objetivos}

La pregunta de investigación es ¿qué criterios mínimos deben cuidar las autoridades nacionales de regulación de la comunicación para garantizar la libertad de expresión y el derecho a la información en el contexto de la pandemia? Se pretende responder desde 1) las orientaciones de organismos internacionales relacionados con la libertad de expresión y los derechos a la comunicación, 2) en comparación con las acciones de reguladores

Bolivia Artículo 14, numeral 21 de la Ley 164 "coor dinar la implementación de las políticas de prevención en los ámbitos de comunicación, información y difusión contra el racismo y toda forma de discriminación y llevar a cabo los procesos sancionatorios" (Gaceta Oficial de Bolivia, 2011).

Colombia Artículo 22 de la Ley 1978. Numeral 25): garantizar el pluralismo e imparcialidad informativa; y, numeral 28): promover y reglamentar lo atinente a la participación ciudadana en los temas que puedan afectar al televidente, especialmente lo referido al control de contenidos audiovisuales (Congreso de Colombia, 2019).

Ecuador Artículo 49 de la Ley Orgánica de Comunicación de Ecuador. El Consejo de Regulación, Desarrollo y Promoción de la Información y Comunicación se encargará de: n) promover iniciativas y espacios de diálogo ciudadanos que coadyuven al ejercicio del derecho a la comunicación (Asamblea Nacional, 2019).

Perú

La Ley 28278 de Radio y Televisión no precisa que las salvaguardas de libertad de expresión, de pensamiento y de opinión sean atribución del Consejo Consultivo de Radio y Televisión, pero están en el marco de la creación del órgano de regulación, particularmente en el artículo II Principios para la prestación de los servicios de radiodifusión, literales b) y e) (Poder legislativo, 2004).

Tabla 1. Marco jurídico. Fuente: Asamblea Nacional del Ecuador, 2019; Congreso de Colombia, 2019; Gaceta Oficial de Bolivia, 2011; Poder legislativo de Perú, 2004. Elaboración propia. 
iberoamericanos, y 3) sobre la base de las publicaciones en medios sociales digitales.

Las autoridades de regulación de comunicación de los países andinos fueron catalogadas (Suing, Ortiz y González, 2016) y corresponden a:

1. Bolivia: Autoridad de Regulación y Fiscalización de las Telecomunicaciones y Transportes ATT.

2. Colombia: Comisión de Regulación de Comunicaciones CRC. (Congreso de Colombia, 2019)

3. Ecuador: Consejo de Regulación, Desarrollo y Promoción de la Información y Comunicación (Asamblea Nacional, 2019).

4. Perú: Consejo Consultivo de Radio y Televisión CONCORTV.

Las bases legales que justifican las intervenciones sobre derechos de comunicación y libertad de expresión están expuestas a continuación (Tabla 1).

\section{Metodología}

El esquema metodológico es cualitativo de tipo descriptivo y relacional (Hernández, Fernández y Baptista, 2000), a través del análisis de contenidos en razón de interpretar textos (Abela, 2013). El enfoque descriptivo evalúa las características de un fenómeno, de acuerdo a Cazau (2006) deben seleccionarse variables para representar de forma detallada el objeto de estudio. A partir de esta caracterización pueden generarse importantes recomendaciones (Abreu, 2012).

El primer análisis de contenidos se realiza a ocho declaraciones sobre COVID-19 de organizaciones y expertos internacionales vinculados con la comunicación y los derechos humanos. Los documentos corresponden a:

1. Estudio sobre las oportunidades de la digitalización en América Latina frente al COVID-19, de la Corporación Andina de Fomento y la Organización de las Naciones Unidas, publicado en abril de 2020 (Agudelo, et al., 2020).

2. Declaración conjunta de la sociedad civil, emitida el 2 de abril de 2020 (Access Now, 2020).

3. Principios para proteger los derechos civiles y políticos en la lucha contra COVID-19, propuestos por Freedom House el 24 de marzo de 2020 (Freedom House, 2020).

4. La declaración conjunta, del 10 de julio de 2019, del Relator Especial de las Naciones Unidas (ONU) para la Libertad de Opinión y de Expresión, el Representante de la Organización para la Seguridad y la
Cooperación en Europa (OSCE) para la Libertad de los Medios de Comunicación, el Relator Especial de la Organización de Estados Americanos (OEA) para la Libertad de Expresión y la Relatora Especial sobre Libertad de Expresión y Acceso a la Información de la Comisión Africana de Derechos Humanos y de los Pueblos (CADHP) (The Centre for Law and Democracy, 2019).

5. La Resolución № 1/2020 "Pandemia y Derechos Humanos en las Américas", adoptada por la Comisión Interamericana de Derechos Humanos el 10 de abril de 2020 (CIDH, 2020).

6. La declaración de los garantes para la libertad de expresión y la libertad de los medios de comunicación de las Naciones Unidas, la Comisión Interamericana de Derechos Humanos y el Representante para la Libertad de los Medios de Comunicación de la Organización para la Seguridad y la Cooperación en Europa, de 19 de marzo de 2020 (OEA, 2020).

7. Las recomendaciones de las organizaciones de la sociedad civil a través del Comité Directivo de Open Government Partnership, del 27 de abril de 2020 (OGP, 2020).

8. I llamado de "Reporteros sin Fronteras" del 27 de abril de 2020 (APM, 2020).

El segundo análisis de contenidos ocurre sobre la información publicada en los sitios Web de las autoridades de regulación de los países andinos, con corte al 11 de mayo de 2020, en comparación con las acciones de varios socios de la Plataforma de Reguladores del Sector Audiovisual de Iberoamérica durante COVID-19 (PRAI, 2019). Las autoridades de regulación consideradas corresponden a dos de América Latina, una de Portugal y otra de España:

- Por Chile, Consejo Nacional de Televisión de Chile CNTV.

- Por España, Consell de l'Audiovisual de Catalunya CAC.

- Por México, el Instituto Federal de Telecomunicaciones de México - IFT.

- Por Portugal, la Entidade Reguladora para a Comunicação Social - ERC.

El último análisis de contenidos se realiza a las publicaciones en medios sociales digitales con corte a mayo de 2020, al mismo tiempo se presenta la gráfica de casos confirmados de COVID-19 por millón de habitantes. 


\section{Resultados}

Los temas en los que coinciden los documentos emanados por las organizaciones y los expertos internaciones son: salud humana, tecnologías de vigilancia digital invasiva, el papel de las empresas, las políticas de telecomunicaciones, las medidas tomadas por los gobiernos para frenar la expansión del COVID-19, y las responsabilidades de los gobiernos y los Estados.

De acuerdo a los documentos, para cuidar la salud de las personas es necesario acceder a Internet y a información precisa sobre la naturaleza del virus, se pide conocer los medios para proteger a las familias y a las comunidades. El derecho a la información debe estar vigente porque es inherente a la salud.

Las tecnologías de vigilancia diseñadas para contener la expansión del COVID-19 deben administrarse de manera abierta, transparente y estar sujetas a supervisión independiente. Se pide que los datos de los ciudadanos sean almacenados durante la emergencia con el único fin de combatir la expansión del virus, así mismo se recomienda evitar discriminaciones que deriven en inadecuadas representaciones de las minorías en los macro conjuntos de datos.

Las empresas están llamadas a respetar los derechos humanos, proporcionar información fiable e intervenir en mitigar el COVID-19 privilegiando el interés público.

Las medidas gubernamentales que implican restricciones a las libertades de los ciudadanos deben tener fundamento legal, promulgarse de manera transparente, es decir compatibles con los estándares internacionales de derechos humanos, ser necesarias y proporcionales frente a la amenaza para proteger la salud, tener duración limitada, prolongarse sobre la base de criterios transparentes y estar sujetas a supervisión independiente.

En atención de los criterios de organizaciones y expertos internacionales, en relación a las competencias de regulación de la comunicación, es responsabilidad de los Estados:

- Respetar y garantizar los derechos humanos, incluye la libertad de expresión.

- Garantizar la privacidad de los ciudadanos, velar porque la recopilación, conservación y agregación de datos personales sea exclusivamente para responder al COVID-19.

- Proteger la libertad de los medios de comunicación y el libre flujo de información.
- Asegurar el derecho de los ciudadanos a acceder a la información pública.

- Menguar los riesgos de discriminación que perjudiquen a las comunidades.

Por su parte, los gobiernos deben proteger la vida de los ciudadanos; entregar información clara, precisa y actualizada sobre COVID-19; garantizar el acceso y abstenerse de bloquear Internet a toda la población y procurar reducir la brecha digital; abstenerse de bloquear sitios de medios de comunicación o ejercer censura previa; y proteger el trabajo de los periodistas y respetar sus fuentes.

Las acciones de política pública y regulatoria tomadas por los gobiernos andinos para enfrentar el COVID-19 (Agudelo, et al., 2020; Blackman, et al., 2020; OGP, 2020; Observacom, 2020; AccessInfo, 2020), con corte al 24 de abril de 2020, son:

\subsection{Bolivia}

1. Protección de personas vulnerables: 1) Transferencia excepcional para familias beneficiarias del "Bono Familia"; 2) Reducción del precio de la energía eléctrica y prohibición de su suspensión por no pago; y, 3) Control de precios para bienes básicos.

2. Medidas fiscales, financieras y monetarias de emergencia: 1) Aplazamiento del pago de impuestos; 2) Moratoria temporal en pago de intereses y préstamos; $y, 3$ ) Crédito de emergencia para PYMES.

3. Medidas de contención: 1) Confinamiento nacional obligatorio; 2) Horario de salida para compras definido según el último dígito de cédula de ciudadanía; 3) Extensión de la cuarentena en diferentes fechas; 4) Uso de tobilleras y manillas electrónicas para hacer seguimiento a personas diagnosticadas o sospechosas; 5) Cierre de colegios y universidades; $y, 6$ ) Restricciones y cierre total de fronteras.

4. Iniciativas ciudadanas: recolectar datos geo-referenciados de abastecimiento, servicios y salud en el contexto de la crisis que luego se distribuyan en formato abierto y sean utilizados por desarrolladores para crear aplicaciones informativas (API estática de JSON) o analistas (en CSV).

5. Libertad de expresión: la Relatoría de Libertad de Expresión de la Comisión Interamericana de Derechos Humanos alertó sobre el desproporcionado uso 
del derecho penal para criminalizar expresiones de interés público.

\subsection{Colombia}

1. Políticas públicas: 1) Ministerio de Educación en conjunto con Sistema de Medios Público RTVC transmiten contenidos educativos, disponibles para descargar en RTVCPlay; 2) Ministerio de Salud emplea canales virtuales para resolver dudas, solicitudes, consultas y trámites. Habilitó aplicación con información de COVID-19; y, 3) Gobierno lanzó página para autodiagnóstico y evitar colapso de líneas de atención u hospitales.

2. Protección de vulnerables: 1) Transferencia extraordinaria beneficiarios de "Familias en Acción" y "Jóvenes en Acción"; 2) Ampliación de cupos de programa "Adulto Mayor"; 3) Reconexión gratuita de servicios públicos a hogares; y, 4) Anticipo de devolución del impuesto IVA a familias en pobreza extrema.

3. Medidas fiscales, financieras y monetarias: 1) Eliminación de aranceles a sector de salud y trasporte aéreo de pasajeros y carga; 2 ) Prórroga de pagos de impuestos a la renta e IVA de personas naturales y empresas de transporte aéreo, hotelero y actividades teatrales y espectáculos; 3) Prórroga de pago contribución parafiscal a promoción del turismo; 4) Línea de crédito para pago nómina de pequeñas empresas; 5) Refinanciamiento, moratoria temporal sobre deudas y créditos de emergencia para PYMES, deudas y moratoria temporal de individuos; 6) Aumento gasto de inversión; 7) Intervención del mercado cambiario; 8) Banco Central redujo la tasa de interés en 50 puntos básicos a 3.75\%.

4. Medidas de contención: 1) Orden de confinamiento nacional. Aislamiento obligatorio de mayores de 70 años. Cierre de colegios y universidades. Transporte público opera en capacidad reducida; 2) Cierre de frontera terrestre, fluvial y marítima, y aérea. Restricción al desplazamiento interno de personas.

5. Iniciativas ciudadanas: gobierno local y organizaciones de sociedad civil unieron esfuerzos para Hackaton hacia soluciones al desafío de movilizar a trabajadores, al tiempo de reducir contagios.

6. Libertad de expresión: 1) Comisión de Regulación de las Comunicaciones recomendó uso responsable de Internet; 2) Presidente Duque decidió (Decreto 516) reducir porcentaje asignado a producciones nacionales en televisión abierta al 20\%; 3) Decreto 491 aplica a derecho a la información, establece que notificación de actos administrativos se hará electró- nicamente, también extiende plazos para solicitudes administrativas.

\subsection{Ecuador}

1. Políticas públicas: 1) Ministerio de Salud habilitó un portal Web con información oficial y acceso a servicios públicos de telemedicina, ubicación de fallecidos, datos abiertos sobre comportamiento del virus, información psicológica, voluntariado en salud, test en línea, entre otros; 2) Ministerio de Telecomunicaciones con Ministerio de Salud Pública presentaron App gratuita "SaludEc" orientada a evaluar síntomas, registrar a ciudadanos para remitir información oficial, constituir canal de telemedicina y de acceso para citas médicas.

2. Protección de vulnerables: 1) Transferencias monetarias excepcionales por dos meses a familias afiliadas a Seguro Social Campesino; 2) Aplazar aportes a seguridad social para afiliados voluntarios y trabajadores independientes; 3) Reconexión obligatoria y control de precios para servicios públicos.

3. Medidas fiscales, financieras y monetarias de emergencia: 1) Reestructuración deudas de gobiernos locales pequeños y medianos con Banco de Desarrollo; 2) Prorroga sin recargo para pago de cuotas de créditos personales, micro y créditos de pequeñas empresas y opción de refinanciamiento; 3) Aplazamiento de pago de impuestos; 4) Aumento de impuestos: 5\% sobre el avalúo de vehículos mayores de 20.000 US\$.

4. Medidas de contención: 1) Confinamiento nacional y toque de queda de 14:00 a 5:00, restricción vehicular según la placa, cierre de colegios y universidades, cancelación de eventos públicos y actividades comerciales no necesarias, transporte público suspendido; 2) Cierre total de fronteras.

5. Iniciativas ciudadanas: sociedad civil desarrolló plataformas de monitoreo para visualizar datos sobre pruebas, evolución curva de aprendizaje del contagio, estado y localización de los casos, entre otros. Ecuador se unió a plataforma colaborativa frenalacurva.net que registra iniciativas ciudadanas frente a COVID-19, formando un repositorio de acceso libre.

\subsection{Perú}

1. Políticas públicas: 1) Ministerio de Transportes y Comunicaciones anunció donación de dos mil tabletas para Ministerio de Salud y garantiza que dispositivos electrónicos cuenten con Internet; 2) Ministerio de 
Salud habilitó plataforma de emergencia 113, procesará ochenta mil llamadas diarias de tele-consulta.

2. Protección a vulnerables: 1) Transferencia excepcional a hogares en pobreza extrema ubicados en zonas de vulnerabilidad sanitaria; 2) Subsidios de incapacidad laboral por 20 días para pacientes confirmados de COVID-19 de ingreso mensual menor a $2.400 \mathrm{~S} /$. (Soles); 3) Creación red apoyo al adulto mayor a través del Ministerio de Inclusión y Desarrollo Social; 4) Reconexión obligatoria y control de precios para servicios públicos.

3. Medidas fiscales, financieras y monetarias de emergencia: 1) Creación de fondo de apoyo a Pymes para garantizar créditos de capital de trabajo, reestructurar y refinanciar sus deudas; 2) Prórroga para declaración del impuesto a la renta de personas naturales y micro, pequeñas y medianas empresas; 3) Reducción de aranceles (cero) a medicamentos y productos médicos; 4) Moratoria temporal en pago de intereses y préstamos; 5) Banco Central reduce tasa de interés en 100 puntos básicos a 1,25\%.

4. Medidas de contención: 1) Orden nacional de aislamiento social obligatorio. Toque de queda entre 18:00 y 5:00. Horario semanal para compras necesarias por género. Cierre de colegios y universidades. Cancelación de eventos públicos y cierre de establecimientos públicos; 2) Cierre total de fronteras aéreas, marítimas, fluviales y terrestres. Restricción a movimiento interno vía terrestre o fluvial de personas.

5. Iniciativas ciudadanas: Municipalidad Metropolitana de Lima, entre otras labores, 1) Implementó mesa virtual para recepción de documentos de entidades públicas vía en línea, relacionados con COVID-19; 2) Presentó "SISOL 40" para asesoramiento médico mediante teléfono o mensajes de WhatsApp; 3) Personas mayores de 60 años con enfermedad crónica reciben atención de un asesor que se comunica a través de plataforma "SISOL a tu lado"; 3) Servicio de orientación psicológica en línea "Lima te escucha"

\begin{tabular}{|c|c|c|c|c|c|c|}
\hline & \multirow{2}{*}{\multicolumn{2}{|c|}{ Criterios }} & \multicolumn{4}{|c|}{ Autoridades nacionales } \\
\hline & & & \multirow{2}{*}{ 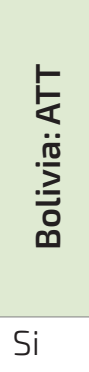 } & \multirow{2}{*}{ 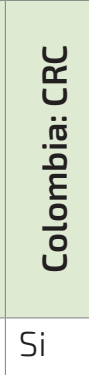 } & \multirow{2}{*}{ 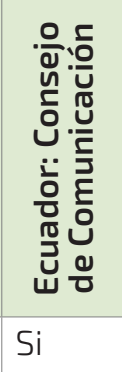 } & \multirow{2}{*}{ 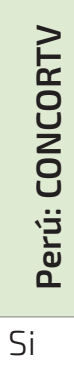 } \\
\hline 1 & \multirow{5}{*}{$\begin{array}{l}\text { Acciones comunes } \\
\text { de los reguladores } \\
\text { iberoamericanos en } \\
\text { COVID-19 }\end{array}$} & Informativas & & & & \\
\hline 2 & & De regulación & $\mathrm{Si}$ & No & $\mathrm{Si}$ & $\mathrm{Si}$ \\
\hline 3 & & De asesoría & No & No & $\mathrm{Si}$ & No \\
\hline 4 & & Concertadas con otros reguladores & No & No & No & No \\
\hline 5 & & Proactivas & No & No & No & $\mathrm{Si}$ \\
\hline 6 & \multirow{5}{*}{$\begin{array}{l}\text { Acciones sugeridas } \\
\text { por organizaciones } \\
\text { y expertos inter- } \\
\text { nacionales } \\
\text { especialistas en } \\
\text { derechos humanos } \\
\text { frente a COVID-19 }\end{array}$} & Garantizar derechos humanos (libertad de expresión) & $\mathrm{Si}$ & $\mathrm{Si}$ & $\mathrm{Si}$ & $\mathrm{Si}$ \\
\hline 7 & & Garantizar la privacidad de ciudadanos & $\mathrm{Si}$ & No & $\mathrm{Si}$ & No \\
\hline 8 & & $\begin{array}{l}\text { Proteger la libertad de los medios de comunicación y el } \\
\text { libre flujo de información }\end{array}$ & $\mathrm{Si}$ & $\mathrm{Si}$ & $\mathrm{Si}$ & $\mathrm{Si}$ \\
\hline 9 & & $\begin{array}{l}\text { Asegurar derecho de los ciudadanos a acceder a informa- } \\
\text { ción pública }\end{array}$ & No & $\mathrm{Si}$ & No & $\mathrm{Si}$ \\
\hline 10 & & Menguar riesgos de discriminación & Si & No & No & Si \\
\hline \multicolumn{3}{|c|}{ Cantidad de criterios que se cumplen } & 6 & 4 & 6 & 7 \\
\hline
\end{tabular}

Tabla 2. Actuaciones de autoridades de regulación del área andina. Fuente: Sitios Web de las autoridades de regulación. Elaboración propia. 


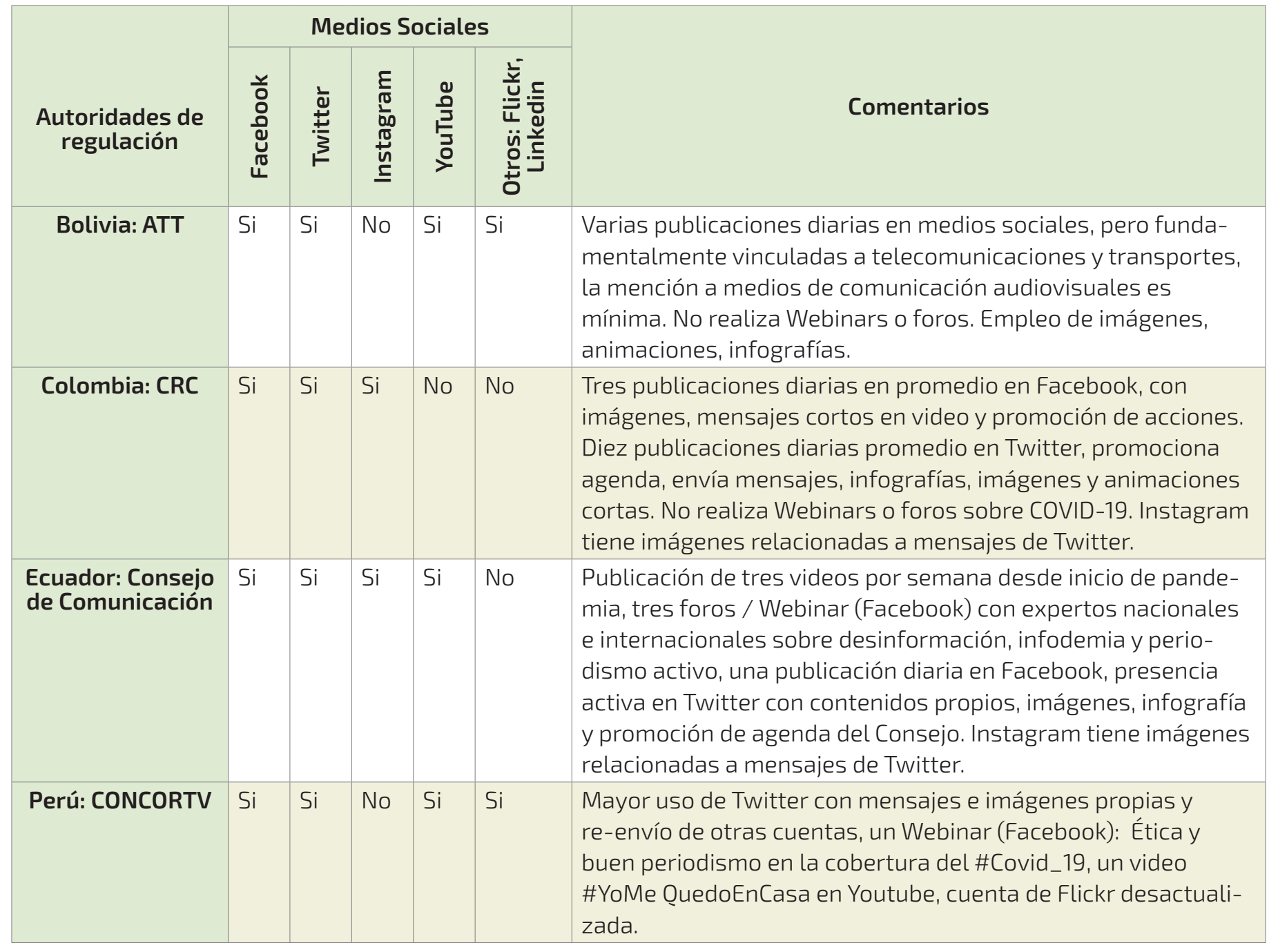

Tabla 3. Contenidos en medios sociales sobre COVID-19. Al 13 de mayo de 2020. Fuente: Cuentas en medios sociales digitales de las autoridades de regulación. Elaboración propia.

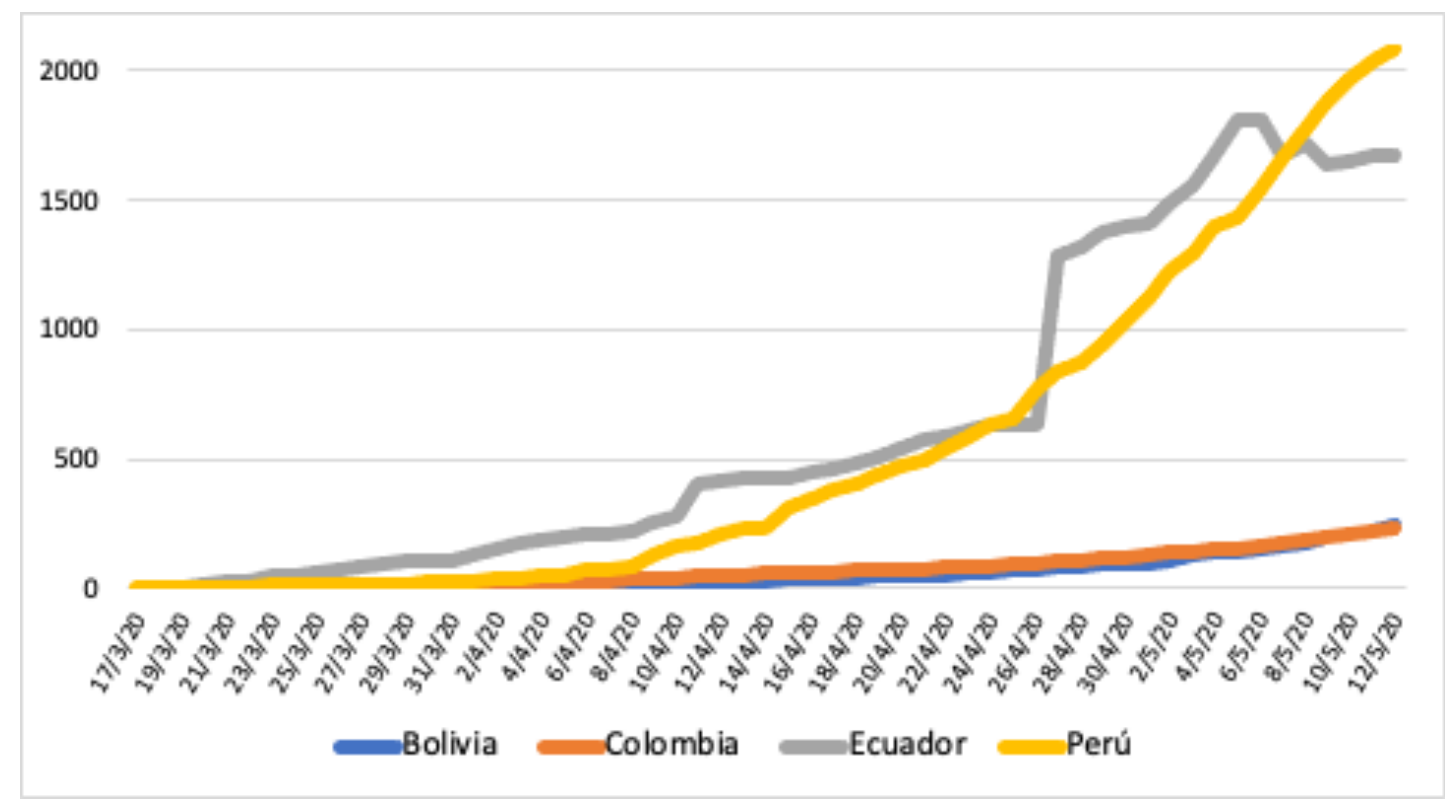

Gráfico 1. Casos confirmados de COVID-19 por millón de habitantes al 12 de mayo de 2020. Fuente: European Centre for Disease Prevention and Control. Elaboración propia. 
para ayudar a sobrellevar el impacto del aislamiento social obligatorio.

Para relacionar las acciones de las autoridades de regulación de los países andinos con las internacionales, primero se presentan las coincidencias entre el CNTV (Chile), CAC (España), IFT (México) y ERC (Portugal):

1. Acciones informativas como boletines, orientaciones para los ciudadanos e instituciones, emisión de cápsulas informativas, constitución de observatorio o micro sitio Web dedicados a COVID-19, alertas para detección de noticias falsas, generación de estadísticas de medios tradicionales y medios sociales.

2. Acciones de regulación a través de la emisión de políticas en materia del audiovisual o las telecomunicaciones.

3. Acciones de asesoría: legal, publicación de recursos y orientaciones para los operadores de medios de comunicación social, empresas de telecomunicaciones y para los usuarios en COVID-19.

4. Acciones concertadas con otros reguladores de la región que se muestran en los sitios Web.

5. Acciones proactivas para emitir espacios educativos para niños y jóvenes a través de la televisión abierta, impulso para generar programas de entretenimiento audiovisual orientados a las familias, y la emisión de alertas sobre periodistas en riesgo.

En la tabla 2 constan los resultados de la confrontación de acciones de los reguladores andinos con los reguladores iberoamericanos, y con las acciones sugeridas a los Estados por parte de organizaciones y expertos internacionales, que en las estructuras políticas de cada nación les corresponden a las autoridades de regulación de la comunicación. La tabla 3 presenta información de los reguladores andinos en medios sociales digitales. Finalmente, el gráfico 1 muestra con fines descriptivos los casos confirmados de COVID-19 entre el inicio de las medidas de aislamiento social y el 12 de mayo de 2020.

\section{Conclusiones}

Del análisis de las declaraciones se desprende que la vigilancia digital propuesta para monitorizar a los contagiados no puede inobservar los acuerdos internacionales de derechos humanos, por lo tanto, la compilación y preservación de datos de las personas con COVID-19 debe complementarse con gestiones para prevenir fraudes, para ello existen leyes de acceso a la información pública.

Lo que señala la Corte Constitucional de Ecuador sobre la vigilancia de los ciudadanos está en armonía con las premisas de órganos internacionales respecto a la privacidad y el uso de datos, pero ésta información es vital para los sistemas de salud, surge entonces un debate sobre derechos humanos y ciencia en COVID-19, en el futuro inmediato las organizaciones, periodistas e investigadores requerirán identificar protocolos de acceso a los datos "reconociendo que será necesario garantizar la privacidad" (CDT, 2020).

Las recomendaciones que brindan los organismos y expertos internacionales coinciden con las premisas que motivaron la creación de órganos de regulación en comunicación: proteger los derechos e intereses individuales, acceso a los medios, diversidad y pluralismo, así mismo guardan coherencia con principios de transparencia, publicidad y gobierno abierto del derecho a la información.

Los Estados respetan y garantizan los derechos humanos, procuran fomentar la libertad de los medios de comunicación y el libre flujo de información. En COVID19 se logra afinar el cumplimiento de los derechos a la comunicación sobre la base de rescatar valores fundamentales de convivencia. Pese a las restricciones de la limitación de movilidad y reunión las medidas adoptadas para frenar los contagios de COVID-19 no socaban los logros conseguidos en la democratización de la comunicación, pero aún falta avanzar hacia la pluralidad y un modelo más democrático en los sistemas mediáticos del área andina, según la clasificación de Hallin y Mancini (2008).

La libertad de expresión es una condición de la democrática por lo que su restricción podría disminuir el progreso sustentable de las naciones andinas. En la construcción de equilibrios entre el control de la pandemia, la recuperación económica y el mantenimiento de las garantías de los ciudadanos cabe acoger los lineamientos de las organizaciones y expertos internacionales en el sentido de resguardar la libertad de los medios de comunicación y articular medidas para el libre flujo de información de manera universal, es decir que cubran más áreas geográficas e incluyan a las nacionalidades, idiomas y culturas que habitan en los países andinos. Esta función está asignada jurídicamente a las autoridades de regulación, como se muestra en la tabla 1.

Sobre la base de las experiencias internacionales en COVID-19, la regulación en comunicación ayuda para promover la información oportuna y de calidad que anime a la salud pública, para esto se realizan acciones 
informativas, de regulación, asesoría, colaborativas y proactivas (tabla 2 ) de parte de reguladores iberoamericanos que no son comunes en los países andinos, por lo tanto, se evidencia una contradicción entre el espíritu de la Comunidad Andina y las prácticas de sus instituciones, es decir, el discurso de integración y colaboración no se pone en práctica por parte de los reguladores de comunicación de Bolivia, Colombia, Ecuador y Perú. El gráfico 1 muestra la importancia de contribuir con información ante las tendencias crecientes de contagios.

En el periodo observado no se realizaron actividades concertadas. Se aprecia un sesgo normativo, un espíritu de sanción en lugar de políticas "blandas" o positivas de los reguladores andinos. Las actividades de apoyo para los operadores y usuarios, y las de formación como la creación de canales web son inexistentes, por lo tanto, hay camino por recorrer a partir de experiencias cercanas y en asociación con plataformas de reguladores internacionales.

Las primeras acciones de los reguladores de la comunicación de los países andinos, publicadas en sus sitios Web, tiene un carácter reactivo, son necesarias y oportunas para informar y articular el trabajo de los medios de comunicación con las políticas nacionales de telecomunicaciones, por ejemplo, la ATT de Bolivia dispuso el 21 de marzo de 2020 la suspensión de los sumarios regulatorios de las telecomunicaciones y la comunicación, pero en general se anticipan muy poco a los escenarios que pueden ocurrir. Es probable que las creaciones recientes de los órganos de regulación incidan en la falta de actividades concertadas, a diferencia de Europa o Norteamérica donde las autoridades de comunicación y del audiovisual tienen más de una década de funcionamiento.

Llama la atención que los reguladores andinos tengan poca actividad en las redes sociales digitales por el alcance e impacto de las mismas, además porque son espacios donde surgen discusiones hacia la concepción de estándares de moderación de los discursos de odio y de la privacidad. La ausencia de publicaciones en medios digitales no implica que los reguladores andinos estén ausentes del tratamiento de los derechos en el espacio digital, pero denota desaprovechar una oportunidad para cumplir con sus misiones ya que, por ejemplo, "los tuits basados en evidencia científica y los de verificación de hechos captan más atención que los de simples hechos" (Pulido, Villarejo-Carballido, Redondo-Sama y Gómez 2020, p. 2).

En la sociedad digital las empresas de telecomunicaciones e Internet son "actores fundamentales para asegurar -o vulnerar- la libertad de expresión (...), en buena medida de ellas depende que las voces críticas puedan ser escuchadas o silenciadas" (Boulin, 2020, p. 41). Los medios sociales digitales a más de la vinculación directa e inmediata que permiten a los ciudadanos, se constituyen en grandes conglomerados que concentran la comunicación, pero no dialogan con los actores nacionales ni pretenden sujetarse a nacientes reglas para el fomento de las economías nacionales.

El detalle de las acciones de política pública y regulatoria muestra actividades de los gobiernos andinos para proteger a las personas débiles, ejemplo de esto son los bonos para las familias y los jóvenes, la reducción de tarifas de los servicios públicos o la devolución de impuestos al valor agregado a las familias pobres. Se desplegaron recursos para que los individuos acojan las restricciones durante los primeros meses de confinamiento, pero hay otras acciones que llevan a "ocultar" información y limitar el trabajo de los medios de comunicación y los periodistas.

En momentos en que es imprescindible contar con información y datos para evitar incertidumbres y violencia algunos líderes andinos pretenden negar los hechos obstaculizando las voces de los periodistas. Emmanuel Colombié, director de la organización Reporteros sin Fronteras (RSF), para América Latina señaló que en Ecuador notaron dificultades para el acceso a la información pública (Escobar, 2020), en Colombia, el gobierno emitió el Decreto Presidencial 491 que extendió los plazos para responder a los pedidos de acceso a la información (Diario Oficial, 2020).

El 13 de julio de 2020, la Federación de Periodistas de América Latina y el Caribe denunció los "despidos masivos" de trabajadores de prensa. En Ecuador, 35 trabajadores del periódico La Hora fueron despedidos (Fundamedios, 2020). En Colombia, el Grupo Semana despidió a 250 periodistas (W Radio, 2020). Así mismo, se detectan restricciones desde las empresas, varios medios de comunicación no proveen equipo protector. En Perú, 37 periodistas murieron por la enfermedad (Efecto Cocuyo, 2020), en Bolivia, el gobierno adoptó disposiciones que tendían a silenciar el discurso legítimo (Human Rights Watch, 2020).

A partir de la pregunta de investigación se buscó identificar los criterios mínimos que deben cumplir las autoridades de regulación de la comunicación de los países andinos para garantizar la libertad de expresión y el derecho a la información en la pandemia. La respuesta específica está en la tabla 2 . Las normas nacionales acogen los debates internacionales sobre pluralismo, diversidad y derecho a la comunicación, y los colocan en las leyes de creación de los reguladores, por lo tanto, no hay ausencia de normas jurídicas (tabla 1), ni de estructuras públicas, lo urgente es poner en marcha 
las ordenaciones diseñadas con la mayor participación posible de los ciudadanos.

En el giro hacia "la nueva normalidad" hay horizontes que los reguladores están convocados a cumplir y que deben ser tratados en futuras investigaciones. El COVID19 presenta desafíos para las instituciones en pro de las libertades y garantías de los ciudadanos. El debate sobre la regulación migra a escenarios digitales ¿cómo hacer uso responsable de las libertades en Internet? En el mundo post - COVID-19 deben existir garantías para la información y comunicación a partir del respeto de los deberes y derechos de las personas, y del cuidado de los bienes comunes.

\section{Referencias}

Abela, J. (2013). Las técnicas de Análisis de Contenido: Una revisión actualizada. http://mastor.cl/blog/wp-content/uploads/2018/02/ Andreu.-analisis-de-contenido.-34-pags-pdf.pdf

Abreu, J. (2012). Hipótesis, método \& diseño de investigación. Daena: International Journal of Good Conscience, 7(2), 187-197. http://www. spentamexico.org/v7-n2/7(2)187-197.pdf

Access Now (2020) Los Estados deben respetar los derechos humanos al emplear tecnologías de vigilancia digital para combatir la pandemia. Access Now. https://www.accessnow.org/cms/assets/ uploads/2020/04/MAR20-GL052_Joint-statement-COVID-19-andsurveillance_ES1.pdf

AccessInfo (2020) COVID-19 Tracker. https://www.rti-rating.org/ COVID-19-tracker/

Agudelo, M.; Chomali, E.; Suniaga, J.; Núñez, G.; Jordán, V.; Rojas, F.; Negrete, J.; Bravo, J.; Bertolini, P.; Katz, R.; Callorda, F., y Jung, J. (2020). Las oportunidades de la digitalización en América Latina frente al COVID-19. CAF, ONU, https://repositorio.cepal.org/bitstream/ handle/11362/45360/4/OportDigitalizaCOVID-19_es.pdf

Aguilar, Ó. (2016). ¿Regular o competir? El caso de la Ley de Servicios de Comunicación Audiovisual de la República Argentina y el principio de proporcionalidad como criterio delimitador. Derecho PUCP, n. 76, pp. 305-338.

Ahmed, W; Vidal-Alaball, J; Downing, J., y López, F. (2020). "COVID-19 and the 5G Conspiracy Theory: Social Network Analysis of Twitter Data". J Med Internet Res, 22(5), e19458 DOI: 10.2196/19458

APM (2020). RSF Pide a la ONU y a la OMS que hagan un llamamiento conjunto en pro de la libertad de prensa. Asociación de Prensa de Madrid. https://wwww.apmadrid.es/rsf-pide-a-la-onu-y-a-la-oms-que-hagan-un-llamamiento-conjunto-en-pro-de-la-libertad-de-prensa/?fbclid=IwAR2IGd75LrhdjCNRRRu03eTWd3zeECRZ5NXo407Lx-gg9wqbmNQ̨zInWZSzw

Asamblea Nacional (20 febrero 2019). "Ley Orgánica de Comunicación". Registro Oficial, Suplemento 432, de 20 de febrero del 2019 http://www.consejodecomunicacion.gob.ec/wp-content/uploads/ downloads/2019/02/Ley_Organica_Comunicacion_reformada.pdf

Bajaña, I. (1 mayo 2020). Incidencias del COVID-19 en Ecuador. Question/Cuestión. e321. https://doi.org/10.24215/16696581e321

Balaguer, L. (2013). Derecho de la información y de la comunicación. Editorial Tecnos.
Bedoya, A. (2020). Lucha por el derecho al anonimato. Elpais.com. https://elpais.com/especiales/2020/coronavirus-COVID-19/predicciones/lucha-por-el-derecho-al-anonimato/

Bizberge, A. (2020). Medios y COVID 19 en América Latina. Prensa CDP http://prensacdp.multisite.rio20.net/medios-y-COVID-19-en-america-latina/

Blackman, A.; Ibáñez, A.; Izquierdo, A.; Keefer, P.; Moreira, M.; Schady, N., y Serebrisky, T. (2020). La política pública frente al Recomendaciones para América Latina y el Caribe COVID-19. BID https://publications.iadb.org/es/la-politica-publica-frente-al-COVID-19-recomendaciones-para--america-latina-y-el-caribe

Boulin, I. (2020) Empresas de telecomunicaciones y libertad de expresión. En FOPEA (2020). Cuando aumentan las necesidades, son aún más importantes las libertades Los desafíos para la libertad periodística en la Argentina en la era de la pandemia, las fake news y el lawfare. FOPEA: https://www.fopea.org/librofopea/

Calo, R. (9 abril 2020). Written Testimony of Professor Ryan Calo. Senate Committee on Commerce, Science, and Transportation. http:// cyberlaw.stanford.edu/files/publication/files/Ryan\%20Calo\%20 Testimony.pdf

CAN (2020). ¿Qué es la Comunidad Andina? Comunidad Andina.org. http://www. comunidadandina.org/Seccion.. sppx?id=189\&tipo=QUE title=somos-comunidad-andina

Carrillo, M. (1986). Los Consejos de prensa como forma de autocontrol: propuestas y prevenciones respecto a su viabilidad en España. Revista de estudios políticos, (54), 77-104

Cazau, P. (2006). Introducción a la Investigación en Ciencias Sociales (3ra. Edición) https://bit.ly/1PPL4Ai

CDT (2020). COVID-19 Content Moderation Research Letter Center for Democracy \& Technology. CDT.org. https://cdt.org/wp-content/ uploads/2020/04/COVID-19-content-moderation-research-letter-PDF-Espanol.pdf

Cetina, C. (2020). Tecnología para la integridad en tiempos del COVID19. CAF. https://scioteca.caf.com/handle/123456789/1542

Chan, A.; Nickson, C.; Rudolph, J.; Lee, A., y Joynt, G. (2020). Social media for rapid knowledge dissemination: early experience from the COVID-19 pandemic. Association of Anaesthetists. https://doi. org/10.1111/anae.15057

CIDH (2020) Pandemia y Derechos Humanos en las Américas. Comisión Interamericana de Derechos Humanos http://www.oas.org/es/ cidh/decisiones/pdf/Resolucion-1-20-es.pdf

Congreso de Colombia (25 julio 2019). Ley 1978 de 2019. Por la cual se moderniza el sector de las tecnologías de la información y las comunicaciones -tic, se distribuyen competencias, se crea un regulador único y se dictan otras disposiciones. Departamento Administrativo de la Función Pública, https://www.funcionpublica.gov.co/eva/ gestornormativo/norma.php?i=98210

Cortes, M. (2020). Enfermedad por coronavirus 2019 (COVID-19): Importancia de la comunicación científica y de la enseñanza actualizada de las zoonosis. Revista peruana de investigación en salud, 4(2), 87-8. https://doi.org/10.35839/repis.4.2.697

Council of Europe (2020). Respecting democracy, rule of law and human rights in the framework of the COVID-19 sanitary crisis. Information Documents SG/Inf(2020)11 https://rm.coe.int/sg-inf2020-11-respecting-democracy-rule-of-law-and-human-rights-inth/16809e 1740

Cué, F. (15 abril 2020). Castigada por el COVID-19, Guayaquil no tiene 
espacio "ni para vivos ni para muertos. France 24. https://www. france24.com/es/20200414-covid19-ecuador-guayaquil-castigada-espacio-vivos-muertos

Diario Oficial. (2020). Decreto 491 de 2020, año CLV. N. 51270 de 28, MARZO, pp. 4. http://www.suin-juriscol.gov.co/viewDocument. asp?ruta=Decretos $/ 30039011$

Efecto Cocuyo. (1 de julio 2020). Más de 186 periodistas han muerto por COVID-19, la mitad en Latinoamérica. Efecto Cocuyo. https://efectococuyo.com/coronavirus/mas-de-186-periodistas-han-muertopor-covid-19-la-mitad-en-latinoamerica/

Escobar, L. (4 de julio 2020). Emmanuel Colombié, director regional de Reporteros sin Fronteras: La pandemia ha hecho aún más visible censura a periodistas. El Universo. https://www.eluniverso.com/noticias/2020/06/28/nota/7887915/periodismo-riesgos-pandemia-coronavirus

European Centre for Disease Prevention and Control (12 mayo 2020). Statistics and Research. Coronavirus (COVID-19) Cases. https:// ourworldindata.org/covid-cases

Evans, C., Sabucedo, P., y Paz, C. (2020): Supporting practice based evidence in the COVID-19 crisis: three researcher-practitioners' stories. Counselling Psychology Quarterly, DOI: 10.1080/09515070.2020.1779661

Freedom House (2020). Principles for Protecting Civil and Political Rights in the Fight against COVID-19. Freedom House.org. https:// freedomhouse.org/article/principles-protecting-civil-and-political-ri ghts-fight-against-COVID-19

Fundamedios. (1 de mayo 2020). Exempleados de Diario La Hora denuncian despidos masivos injustificados y exigen que se respeten sus derechos laborales. Fundamedios.org. https://www.fundamedios.org.ec/alertas/diario-lahora-guayaquil-covid-despidos/

Gaceta Oficial de Bolivia (8 de agosto 2011). Ley № 164. Ley general de telecomunicaciones, tecnologías de información y comunicación. Gaceta Oficial. https://www.att.gob.bo/content/normas

Hallin, D., y Mancini, P. (2007): Un estudio comparado de los medios en América Latina. En Díaz, B. (dir.): Tendencias '07. Medios de comunicación en el escenario iberoamericano. Ariel.

Hallin, D., y Mancini, P. (2008): Sistemas mediáticos comparados: tres modelos de relación entre los medios de comunicación y la política. Hacer.

Hernández, M.; Badillo, Á y Peña, M. (2018). Discusión y propuesta metodológica para el análisis comparado de legislaciones audiovisuales generales de carácter nacional. Intercom, 47(3), 1-20. https:// www.redalyc.org/articulo.oa?id=698/69858441003

Hernández, R.; Fernández, C., y Baptista M. (2000) Metodología de la Investigación (5 ed.). McGraw-Hill /Interamericana Editores, S.A.

Human Rights Watch. (7 abril 2020). Bolivia: Decreto sobre Covid-19 Amenaza la Libertad de Expresión. Human Rights Watch. https:// www.hrw.org/es/news/2020/04/07/bolivia-decreto-sobre-covid19-amenaza-la-libertad-de-expresion

Joh, E. (18 marzo 2020). Yes, States and Local Governments Can Close Private Businesses and Restrict Your Movement. Politico.com. https://www.politico.com/news/magazine/2020/03/18/states-police-power-coronavirus-135826

Jurado, R. (2010). Un nuevo paradigma latinoamericano en la regulación de la comunicación. Chasqui, (112), 86-90. https://www.redalyc. org/articulo.oa?id=160/16057456017
Krakowiak, F. (2018). La regulación audiovisual en el Sistema Interamericano de Derechos Humanos y los desafíos de la digitalización. Zer: Revista de Estudios de Comunicación, 23(44), 121-138. https://doi.org/10.1387/zer.18829

Lauría, C. (2020). Libertad de expresión, ¿otra víctima del COVID-19? En FOPEA. Cuando aumentan las necesidades, son aún más importantes las libertades Los desafíos para la libertad periodística en la Argentina en la era de la pandemia, las fake news y el lawfare. FOPEA. https://www.fopea.org/librofopea/

Loreti, D., y Luis L. (2014). El derecho a comunicar. Los conflictos en torno a la libertad de expresión en las sociedades contemporáneas. Siglo XXI.

Loreti, D. (2005). América Latina y la libertad de expresion. Grupo Editorial Norma.

Massaad, E., y Cherfan, P. (2020). Social Media Data Analytics on Telehealth During the COVID-19 Pandemic. Cureus, 12(4), e7838. https:// doi.org/10.7759/cureus.7838

McQuail, D. (2010). Media regulation (module 2, unit 11). Manuscrito inédito en Hernández, Marina; Badillo, Ángel; Peña, María (2018). "Discusión y propuesta metodológica para el análisis comparado de legislaciones audiovisuales generales de carácter nacional". Intercom, 47(3), 1-20. https://www.redalyc.org/articulo. oa?id=698/69858441003

Mendel, T. (2009). El Derecho a la Información en América Latina. Unesco.

Moreno-Montoya, J. (2020). El desafío de comunicar y controlar la epidemia por coronavirus. Biomédica, 40(1), 11-13. https://www. redalyc.org/articulo.oa?id=843/84362871002

Observacom (2020). Iniciativas de los gobiernos y reguladores: TIC y telecomunicaciones servicios esenciales. https://www.observacom. org/acceso-a-Internet-y-gestion-de-redes-de-telecomunicaciones-ante-el-COVID-19-en-america-latina/

OEA (2020). COVID-19: Los gobiernos deben promover y proteger el acceso y la libre circulación de la información durante la pandemia. Comunicado de prensa R58/20. http://www.oas.org/es/cidh/expresion/showarticle.asp?art|D=1170\&lID=2

OGP (2020). Comunicado sobre la respuesta COVID-19 de miembros de la sociedad civil del Comité Directivo de OGP. Open Government Partnership https://www.opengovpartnership.org/es/news/statement-on-the-COVID-19-response-from-civil-society-members-ofogp-steering-committee/

Pimiento, J. (2013). La regulación. Análisis a partir de las funciones jurisdiccionales de la Comisión de Regulación de Comunicaciones. Revista Digital de Derecho Administrativo, (9), 15-40. https:// www. redalyc.org/articulo.oa?id=5038/503856214003

Poder Legislativo (16 julio 2004). Ley 28278 de radio y televisión. El Peruano, 272517. http://www.concortv.gob.pe/es/file/normatividad/2004/NL20040716.pdf

PRAI (2019) Miembros Activos. Plataforma de Reguladores del Sector Audiovisual de Iberoamérica. http://prai.tv/miembros/

Pulido, C.; Villarejo-Carballido, B.; Redondo-Sama, G., y Gómez, A. (2020). COVID-19 infodemic: More retweets for science-based information on coronavirus than for false information. International Sociology, 1-16. https://doi.org/10.1177/0268580920914755

Romero, D. (7 abril 2020). Cuatro parámetros limitan rastreo de 
personas positivas con COVID-19. El Comercio.com. https://www. elcomercio.com/actualidad/parametros-rastreo-personas-positivas-covid19.html

Ruvalcaba, J.; Beltrán, M.; Benítez, A.; Santiago, D.; García, F.; Toribio, J.; Vázquez, J., y Peña, E. (2020). Una reflexión en torno a Atención Primaria en Salud y la pandemia por SARS COVID-2; COVID-19 en México ante la confusión en la comunicación. JONNPR, 5(6), 631-43. https://doi.org/10.19230/jonnpr.3710

Segura, M. (2020). Con alerta pero sin pánico. El rol de los medios durante la pandemia. Revista de la Facultad de Ciencias Médicas de Córdoba, 77(1), 55-58. https://doi.org/10.31053/1853.0605.v77. n1.28066

Stanley, J., y Granick, J. (8 abril 2020). The Limits of Location Tracking in an Epidemic. ACLU. https://www.aclu.org/report/aclu-white-paper-limits-location-tracking-epidemic?redirect=aclu-white-paper-limits-location-tracking-epidemic

Suing, A.; Ortiz, C., y González, V. (2016): Configuración de las autoridades de regulación de la televisión en los países andinos. Revista Latina de Comunicación Social, (71), 730-749. https://doi.org/10.4185/ RLCS-2016-1118

The Centre for Law and Democracy (2019). Declaración conjunta del vigésimo aniversario: desafíos para la libertad de expresión en la próxima década. https://www.law-democracy.org/live/wp-content/ uploads/2019/07/mandates.decl_.2019.20th.Spanish.pdf

Torres, D., y Valenzuela, J. (2020). Lost in Translation: Sesgos y la información en la pandemia de COVID-19. Revista Chilena de Anestesia, 49(3), 401-407. https://doi.org/10.25237/revchilanestv49n03.019

Torres, J. (13 mayo 2020). Piura ya no tiene camas libres en UCI para pacientes graves con COVID-19. Radio Cutivalú. https://www.radiocutivalu.org/piura-ya-no-tiene-camas-libres-en-uci-para-pacientesgraves-con-COVID-19/

Vassoler-Froelich, I. (2020) Entrevista con Andrei Cambronero Torres: Análisis de la pandemia de COVID-19 desde Costa Rica. Middle Atlantic Review of Latin American Studies, 4(1), 5-15.

Véliz, C. (2020). Un mundo con menos pantallas. Elpais.com. https:// elpais.com/especiales/2020/coronavirus-COVID-19/predicciones/ un-mundo-con-menos-pantallas/

Viet-Phuong, L.; Thanh-Hang, P.; Manh-Toan, H.; Minh-Hoang, N.; Khanh-Linh, N.; Thu-Trang, V.; Hong-Kong, N.; Trung, T.; Quy, K.; ManhTung, H., y Quan-Hoang, V. (2020). Policy Response, Social Media and Science Journalism for the Sustainability of the Public Health System Amid the COVID-19 Outbreak: The Vietnam Lessons. Sustainability, v. 12, n. 2931. https://doi.org/10.3390/su12072931

W Radio (8 marzo 2020). Grupo Semana suspende hasta por 60 días ediciones impresas de cinco revistas. W Radio. https://www.wradio. com.co/noticias/actualidad/\%20grupo-semana-suspende-hasta-por-60-dias-ediciones-impresas-de-cinco-revistas/20200318/ nota/4023717.aspx

\section{CV}

Abel Suing. Docente e investigador del Departamento de Ciencias de la Comunicación de la Universidad Técnica Particular de Loja (UTPL) donde actualmente coordina la Maestría en Comunicación mención investigación y cultura digital. Investigador acreditado por Secretaria Nacional de Educación Superior Ciencia, Tecnología e Innovación del Ecuador - REG-INV-18-03088. Licenciado en economía por la UTPL. Doctor en Comunicación por la Universidad de Santiago de Compostela. Coordinador del Grupo de Investigación Comunicación y Cultura Audiovisual (GICA). Integrante de la Redes de investigación INAV y RICE. Socio de la Unión Nacional de Periodistas de Ecuador. Líneas de investigación: Televisión, Políticas de Comunicación, Educación Superior.

Lilia Carpio-Jiménez. Máster en Artes Visuales por la Universidad Nacional Autónoma de México. Licenciada en Arte y Diseño por la Universidad Técnica Particular de Loja. Docente universitario (Establecimiento UTPL) de las asignaturas Diseño Gráfico, Diseño de Comunicación Visual, y Teoría de la Imagen. Experiencia de 8 años de trabajo en el Departamento de Ciencias de la Comunicación de la UTPL. Diseñadora Gráfica en la Gerencia de Marketing de la UTPL durante 6 años. Investiga temas de arte, diseño, fotografía, imagen, redes sociales y género. Es parte del grupo de investigación Comunicación y Cultura Audiovisual y de la Red Internacional de Investigación de Gestión de la Comunicación.

Kruzkaya Ordóñez. Doctora en Comunicación e Información Contemporánea por la Universidad de Santiago de Compostela. Máster en Comunicación e Industrias Creativas. Licenciada en Comunicación. Coordinadora de la Titulación de Comunicación y Docente anexa al Departamento de Ciencias de la Comunicación de la Universidad Técnica Particular de Loja, Ecuador. Integra la sección departamental Narrativas Audiovisuales de la UTPL. Líneas de investigación medios audiovisuales, comunicación digital, divulgación científica, periodismo de investigación. Profesora de pregrado y postgrado. Directora y productora de documentales de investigación científica. Miembro del comité de Periodismo educativo del Ecuador. Participa en la Red de Narrativas Audiovisuales, Red Xescom, RICE. Participa en Proyectos con financiación institucional y externa. 